\title{
Metabolism in tumour-associated macrophages: a quid pro quo with the tumour microenvironment
}

\author{
Xiang Zheng (10 1,6 Siavash Mansouri ${ }^{1,6}$, Annika Krager ${ }^{1}$, Friedrich Grimminger ${ }^{2}$, \\ Werner Seeger (1) ${ }^{1,2,3}$, Soni S. Pullamsetti (1) ${ }^{1,2}$, Craig E. Wheelock $\mathbb{1}^{4}$ and \\ Rajkumar Savai (1) $1,2,3$
}

\begin{abstract}
Affiliations: ${ }^{1}$ Max Planck Institute for Heart and Lung Research, Member of the German Center for Lung Research (DZL), Member of the Cardio-Pulmonary Institute (CPI), Bad Nauheim, Germany. ${ }^{2}$ Dept of Internal Medicine, Member of the DZL, Member of CPI, Justus Liebig University, Giessen, Germany. ${ }^{3}$ Institute for Lung Health (ILH), Justus Liebig University, Giessen, Germany. ${ }^{4}$ Division of Physiological Chemistry 2, Dept of Medical Biochemistry and Biophysics, Karolinska Institute, Stockholm, Sweden. ${ }^{5}$ Frankfurt Cancer Institute (FCI), Goethe University, Frankfurt, Germany. ${ }^{6}$ These authors contributed equally.
\end{abstract}

Correspondence: Rajkumar Savai, Molecular Mechanisms in Lung Cancer, Dept of Lung Development and Remodelling, Max Planck Institute for Heart and Lung Research, Parkstrasse 1, D-61231 Bad Nauheim, Germany. E-mail: rajkumar.savaiampi-bn.mpg.de

@ERSpublications

Tumour-associated macrophages (TAMs) display a high level of functional plasticity and altered metabolism symbolised by high sensitivity to the surrounding tumour microenvironment. The metabolism of TAMs provides novel therapeutic opportunities to treat cancer. https://bit.ly/31OqHhe

Cite this article as: Zheng X, Mansouri S, Krager A, et al. Metabolism in tumour-associated macrophages: a quid pro quo with the tumour microenvironment. Eur Respir Rev 2020; 29: 200134 [https://doi.org/ 10.1183/16000617.0134-2020].

ABSTRACT Lung cancer is the leading cause of death from cancer worldwide. Recent studies demonstrated that the tumour microenvironment (TME) is pivotal for tumour progression, providing multiple targeting opportunities for therapeutic strategies. As one of the most abundant stromal cell types in the TME, tumour-associated macrophages (TAMs) exhibit high plasticity. Malignant cells alter their metabolic profiles to adapt to the limited availability of oxygen and nutrients in the TME, resulting in functional alteration of TAMs. The metabolic features of TAMs are strongly associated with their functional plasticity, which further impacts metabolic profiling in the TME and contributes to tumourigenesis and progression. Here, we review the functional determination of the TME by TAM metabolic alterations, including glycolysis as well as fatty acid and amino acid metabolism, which in turn are influenced by environmental changes. Additionally, we discuss metabolic reprogramming of TAMs to a tumouricidal phenotype as a potential antitumoural therapeutic strategy.

\section{Introduction}

Lung cancer is the most frequently diagnosed cancer and exhibits the highest mortality rate of all cancers worldwide [1, 2]. However, in the last two decades, lung cancer therapy has been revolutionised from old-fashioned cytotoxic chemotherapies to immune checkpoint therapies, leading to a personalised medicine approach in which the tumour microenvironment (TME) is a key determinant of tumour growth and metastasis rather than a bystander $[3,4]$.

Provenance: Commissioned article, peer reviewed

Received: 5 May 2020 | Accepted after revision: 6 Aug 2020

Copyright $\odot$ ERS 2020. This article is open access and distributed under the terms of the Creative Commons Attribution Non-Commercial Licence 4.0. 
The lung TME consists of a heterogeneous cell population that includes cancer cells, stromal cells and immune cells $[3,5]$. Evolutionary game theory has been applied in the context of cancer, which indicates that cancer cells are the dominant tumour cells. Crosstalk between cancer cells and other non-malignant cells demonstrates a pivotal role in tumourigenesis $[6,7]$. Studies from our group clearly demonstrate that tumour-associated macrophages (TAMs) play a central role in lung cancer growth and metastasis, with bidirectional cross-talk between macrophages and cancer cells via the $\mathrm{C}-\mathrm{C}$ chemokine receptor type 2 (CCR2) and CX3C chemokine receptor 1 (CX3CR1) signalling as a central underlying mechanism [5, 8]. In the same line, it has been demonstrated that depletion of TAMs through different approaches such as colony stimulating factor 1 (Csf1) inactivation, CSF1 receptor (CSF1R) antibodies and clodronate liposomes diminishes angiogenesis and tumour growth in different tumour models [9, 10]. Interestingly, the combination of TAM-targeted therapies such as CSF1R inhibition with immune checkpoint therapies not only reduces TAMs infiltration and their immunosuppressive phenotypes, but also improves the response to immune checkpoint therapies by completely impaired tumour growth and even regression of the established tumour [11]. In addition, crosstalk between lung cancer cells and T-helper (Th)9/Th17 lymphocytes plays a major role in lung cancer cell epithelial mesenchymal transition, thereby promoting migration and metastasis [12]. It has been shown that other immune cells such as dendritic cells and neutrophils have functional roles in tumourigenesis. In this line, dendritic cells render an immunosuppressive phenotype to TME by modulation of T-cell differentiation and proliferation through secretion of transforming growth factor- $\beta$ and indoleamine 2,3-dioxygenase (IDO), respectively [13]. Tumour-associated neutrophils are also one of the prominent immune cells within TME that have significant impacts on tumour initiation and progression through production of reactive oxygen species (ROS)/reactive nitrogen species and proteases, which not only support cancer cell transformation but also angiogenesis and metastasis [14]. In the TME, cells demonstrate limited access to oxygen and nutrients, due to disorganised surrounding blood vessels. In this metabolically unstable environment, cells must adapt their metabolic profiles. Therefore, metabolic crosstalk between different cell types for oxygen and nutrients is not only necessary for individual cell survival, but is also a key determinant of cancer cell maintenance and growth and/or modification of the microenvironment to promote cancer cell survival [15].

Metabolic crosstalk between cancer cells and immune cells is involved in the immunosuppression of tumours. Cancer cells produce lactic acid and IDO, which inhibit T-cell proliferation and function. Effector T-cells and cytotoxic T-cells compete with cancer cells to reprogram their metabolic profiles towards glycolysis. However, according to Darwinian fitness, only one winner can exist. Thus, cancer cell survival is primarily due to their pro-tumour phenotype and T-cell exhaustion [3]. Additionally, metabolic crosstalk between cancer cells and regulatory T-cells (Tregs) or myeloid-derived suppressor cells leads to modification of immune cell metabolism, which enables immune cells to use cancer cell metabolites [16].

TAMs are one of the most abundant immune cells in the TME of lung cancer and other types of cancer. The abundance of TAMs in the TME correlates with poor prognosis and disease progression in nonsmall cell lung carcinoma, whereas a higher abundance of TAMs in colorectal cancer is associated with better clinical outcomes $[17,18]$. These clinical studies are consistent with the protumoural and antitumoural functions of TAMs in in vivo and in vitro tumour models. In terms of protumoural function, depletion of TAMs by clodronate liposomes and macrophage fas-induced apoptosis transgenic mice showed decreased tumour growth [8]. In addition, protumoural TAMs contribute to tumour progression by supporting angiogenesis and suppressing antitumoural activity in immune cells [19]. Importantly, a growing body of evidence show that TAMs can reduce the efficiency of immune checkpoint therapies; thus, targeting TAMs can potentiate checkpoint inhibitor therapies [20]. In contrast, antitumoural TAMs can impede tumour development by producing pro-inflammatory cytokines such as tumour necrosis factor (TNF)- $\alpha$ and reactive oxygen intermediates [21]. However, we do not have a clear picture of TAM metabolism during tumour initiation and progression, and we do not know whether the TAM metabolic profile is associated with pro- and/or antitumoural functions, especially in lung cancer.

Lung macrophages are categorised by their embryogenic origin and anatomical location in adult lungs and can include yolk sac-derived primitive interstitial macrophages, fetal liver-derived alveolar macrophages and bone marrow-derived definitive interstitial macrophages. Alveolar macrophages are key players in the phagocytosis of foreign bodies and catabolism of surfactants, whereas interstitial macrophages exhibit functional roles in tissue remodelling, haemostasis and antigen presentation [22]. In cancer, despite the complexity and high plasticity of TAMs, the dichotomy has been used to categorise TAMs into classically activated macrophages (M1) that exhibit antitumoural functions and alternatively activated macrophages (M2) that possess a protumoural phenotype. In the simplified metabolic view, M1 macrophages are often considered highly glycolytic with a broken tricarboxylic acid (TCA) cycle and substantial production of ROS. In contrast, M2 macrophages possess an intact TCA cycle that is coupled to oxidative phosphorylation 
(OXPHOS) for energy production [23]. Although researchers suggested that TAMs are M2-like macrophages, this binary simplified categorisation cannot be indiscriminately applied to the dynamic TME in which TAMs must adopt their metabolic profile based on the availability of oxygen and nutrients [24]. Here, we discuss TAM metabolism and its influence on the TME via crosstalk with cancer cells.

\section{TAM metabolism configures the TME and the TME determines the TAM phenotype Glucose metabolism}

TAM glycolysis is associated with TAM recruitment and tumourigenesis. TAMs are mainly derived from circulating monocytes that infiltrate tumour sites in response to chemoattractants, such as chemokines and proinflammatory signals [25]. TAMs encounter a gradual decline in oxygen during extravasation from the blood vessel, which demonstrates a high oxygen availability, to the TME, which exhibits many hypoxic areas [26]. This capacity to migrate is dependent on glycolysis, because inhibition of macrophage glycolysis by dichloroacetic acid strongly diminishes macrophage migration [27]. In addition, dichloroacetic acid profoundly decreases macrophage migration to implanted matrigels in a lung tumour xenograft model [27]. As cellular migration is energetically demanding during this process, adenosine triphosphate (ATP) production by glycolysis can sustain cytoskeletal remodelling [28]. Consistently, pyruvate kinase muscle2, as the key glycolytic enzyme for ATP production, co-localises with F-actin in macrophage filopodia and lamellipodia, which are involved in macrophage migration [27]. Although the TAM phenotype is unknown in cancer cell initiation, it seems that the glycolytic phenotype is one of the basic instincts of TAMs in the early stages of tumourigenesis. Hence, further studies are needed to address how the glycolytic phenotype of TAMs shapes the TME in the initiation step.

Following the settling of TAMs and tumour establishment, the TME transits to a Th2-type environment in which TAMs are polarised and acquire a protumoural function by being subjected to various factors including interleukin (IL)-4 produced by cancer cells and $\mathrm{CD}^{+}$T-cells, colony-stimulating factor-1, and granulocyte-macrophage colony-stimulating factor produced by cancer cells [29, 30]. Based on the hypoxic and nutrient limitation of the TME, TAMs maintain their glycolytic phenotype after migration. A comprehensive proteomic analysis of tumour extract-stimulated bone marrow-derived macrophages (TES-TAMs) shows upregulation of aerobic glycolysis in comparison with bone marrow-derived macrophages [31]. Moreover, the key regulatory glycolytic enzymes, hexokinase 2 (HK2), phosphofructokinase, and enolase1 (ENO1) are upregulated in mouse mammary tumor virus-polyoma virus middle $\mathrm{T}$ antigen (MMTV-PyMT) murine tumour model-derived TES-TAMs and primary TAMs [31].

Activated TAM glycolysis is also involved in angiogenesis and tumour metastasis [32]. Co-culture experiments on human peripheral blood monocytes with two human cancer cell types, including pancreatic ductal adenocarcinoma (PDAC) cells and medullary thyroid carcinoma cells, reveal a strong glycolytic profile in TAMs with an increase in the transcription of glycolysis-associated genes and a shift towards aerobic glycolysis in an mammalian target of rapamycin (mTOR)-dependent manner, respectively [33, 34]. In these systems, a reduction in the pro-metastatic capabilities of TAMs by HK2 inhibition reveals that the glycolytic profile of TAMs is a necessary tool that determines the metastatic capabilities of cancers. However, astonishing cooperation occurs in the hypoxic areas of the TME, where TAMs reside in large numbers [35]. Hypoxic TAMs exhibit an increased glycolysis rate accompanied by upregulation of growth factors, such as vascular endothelial growth factor (VEGF) and platelet-derived growth factor, which can sustain tumour growth by inducing angiogenesis and metastasis [35]. A high glycolytic profile is considered a protumoural feature of hypoxic TAMs; however, in lung cancer, modification of the mTOR-REDD1 axis in hypoxic TAMs reprogrammes metabolism towards a higher glycolytic state that can reduce the metastatic burden by inducing glucose competition between TAMs and endothelial cells [36]. This means that the high glycolysis rate is precisely fine-tuned in hypoxic TAMs in a way that not only supports the prometastatic capacity of TAMs, but also maintains the glucose concentration in the perivascular space at a level that can induce endothelial hyperactivation and disorganise the tumour vasculature. Interestingly, TAMs also directly contribute to the induction of tumour hypoxia and glycolysis in cancer cells, which could be induced by activation of 5'-adenosine monophosphate-activated protein kinase, which increases glucose uptake and glycolysis flux, and enhances the mitochondrial oxygen consumption rate in TAMs in lung cancer (figure 1) $[37,38]$. Therefore, TAM metabolism can force cancer cells to adopt glycolysis as their primary metabolic pathway, thereby rendering an invasive cancer cell phenotype [39].

Our group recently identified an increase in the transcription of glycolysis-associated genes in fluorescence-activated cell sorting of TAMs from human lung tumours compared with counterparts from matched non-tumour tissues. Intriguingly, the TAM glycolytic gene profile was similar between TAMs derived from invasive margins and tumour central compartments (unpublished data). These data suggest that the glycolytic phenotype of TAMs is an independent variable in the status of the TME. Overall, the glycolytic phenotype of TAMs can be considered an innate feature that confers protumoural functions in 


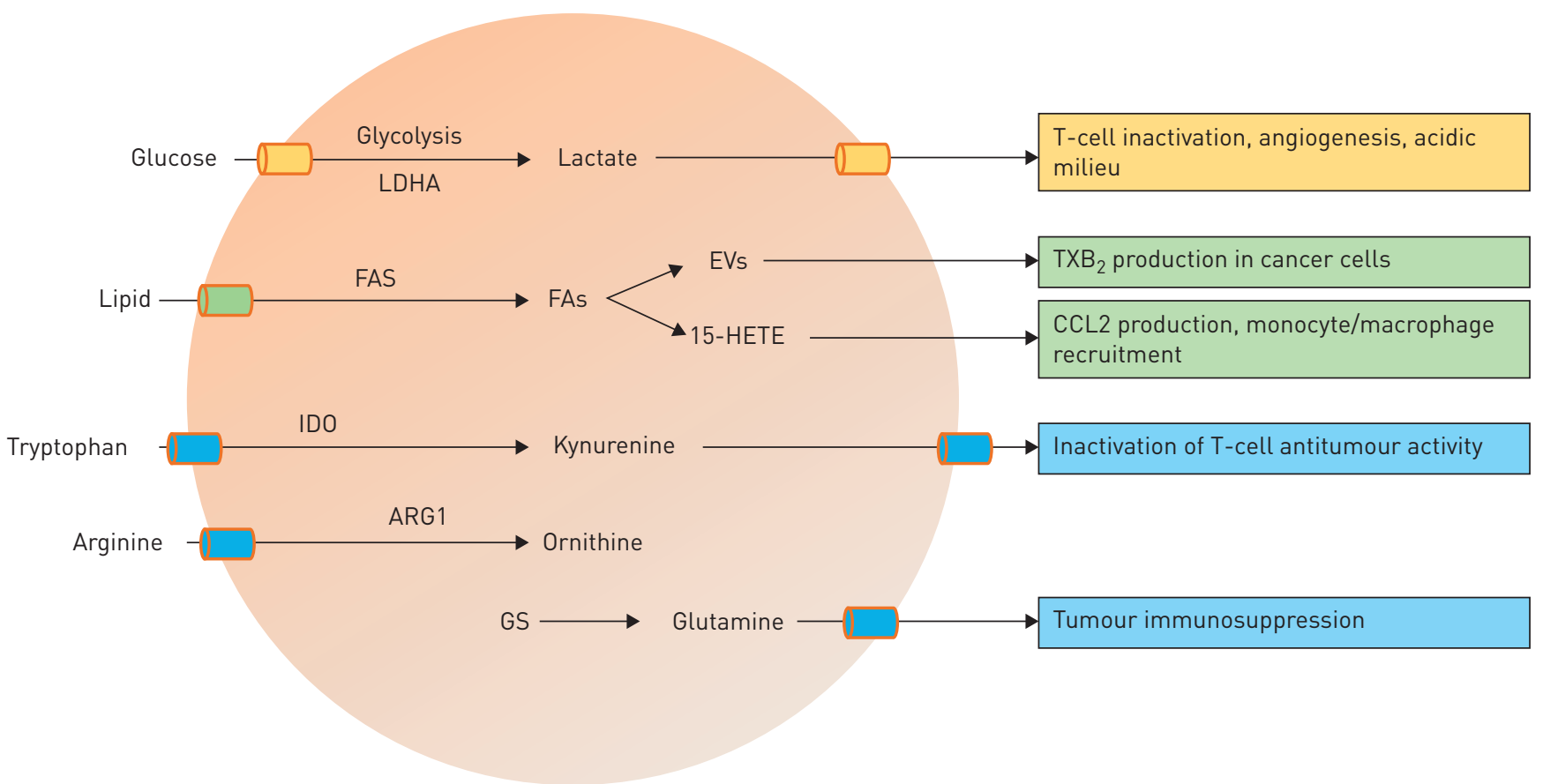

FIGURE 1 Tumour microenvironment (TME) metabolites activate tumour-associated macrophages (TAMs). In TME, glucose, lipid, tryptophan and arginine metabolites activate TAMs. These activated TAMs consume glucose, tryptophan and arginine leading to depletion of these metabolites, which in turn impact TME and its immune status. Moreover, TAM-derived metabolites le.g. lactic acid, kynurenine, glutamine and 15-hydroxyeicosatetraenoic acid (15-HETE)) regulate angiogenesis, T-cell activation and tumour progression. Transporters and/or metabolites of glucose metabolism are indicated as orange, amino acid metabolism as blue and lipid metabolism as green. LDHA: lactate dehydrogenase A; EV: extracellular vesicle; IDO: indolamin-2,3-dioxygenase; ARG: arginase; GS: glutamine synthase; TXB ${ }_{2}$ : thromboxane B2; FAS: fatty acid synthase; FAs: fatty acids.

TAMs, leading to metabolic cooperation with cancer cells and establishment of a pro-invasive TME. However, further investigations into the contribution of TAM glycolysis in recruitment and function of other immune and stromal cells are required.

The high glycolytic rate of tumour cells results in increased production of lactic acid. Tumour cell-derived lactic acid induces hypoxia inducible factor (HIF)- $1 \alpha$-dependent protumoural polarisation of TAMs and hypoxia is associated with accumulation of protumoral TAMs [119]. When exposed to lactic acid-stimulated macrophages, both murine tumour models using LCC1 and B16-F1 melanoma cell lines resulted in larger tumours compared with co-injection of control medium-stimulated macrophages [40]. To promote neovascularisation, lactic acid induces VEGF production in TAMs by stabilising HIF-1 $\alpha$ [40]. Moreover, tumour-derived lactic acid activates mTORC1 to suppress ATP6V0d2-targeted HIF2 $\alpha$ degradation in TAMs, leading to M2 polarisation with enhanced HIF2 $\alpha$-mediated VEGF production [41]. Additionally, lactic acid produced by tumours stimulates IL-23 production in TAMs, resulting in tumour growth by inducing the production of IL-17 and IL-22 [42]. Moreover, the acidic TME results from high glycolysis of tumour cells and poor perfusion is characterised as a pivotal factor in tumour progression. Acidity (independent from lactic acid) augments the protumoural polarisation of TAMs in prostate cancer [43]. Tumour cell-derived lactic acid affects glucose metabolism in TAMs. A human macrophage-thyroid carcinoma cell line co-culture model reveal that thyroid cancer-derived lactic acid induces aerobic glycolysis in TAMs through the AKT1/mTOR pathway [33]. Because HIF-1 $\alpha$ activation is highly dependent on mTOR activation, this study is in line with the finding that lactic acid produced by tumour cells induces a protumoural phenotype in TAMs by inducing HIF-1 $\alpha$ [40]. Apart from the influence of tumour cell-derived lactic acid, enhanced endogenous aerobic glycolysis induced a protumoural phenotype in TAMs. A proteomics analysis demonstrated increased aerobic glycolysis with upregulated HK2, phosphofructokinase and ENO1 in TAMs (figure 2) [31]. Furthermore, increased aerobic glycolysis in TAMs is linked to invasion and metastasis of PDAC [34]. Recently, researchers showed that cancer cell-derived succinate triggers the PI3K-HIF-1 $\alpha$ axis in macrophages by activating the succinate receptor, polarising macrophages into protumoural TAMs that induce cancer cell migration and metastasis [44]. 


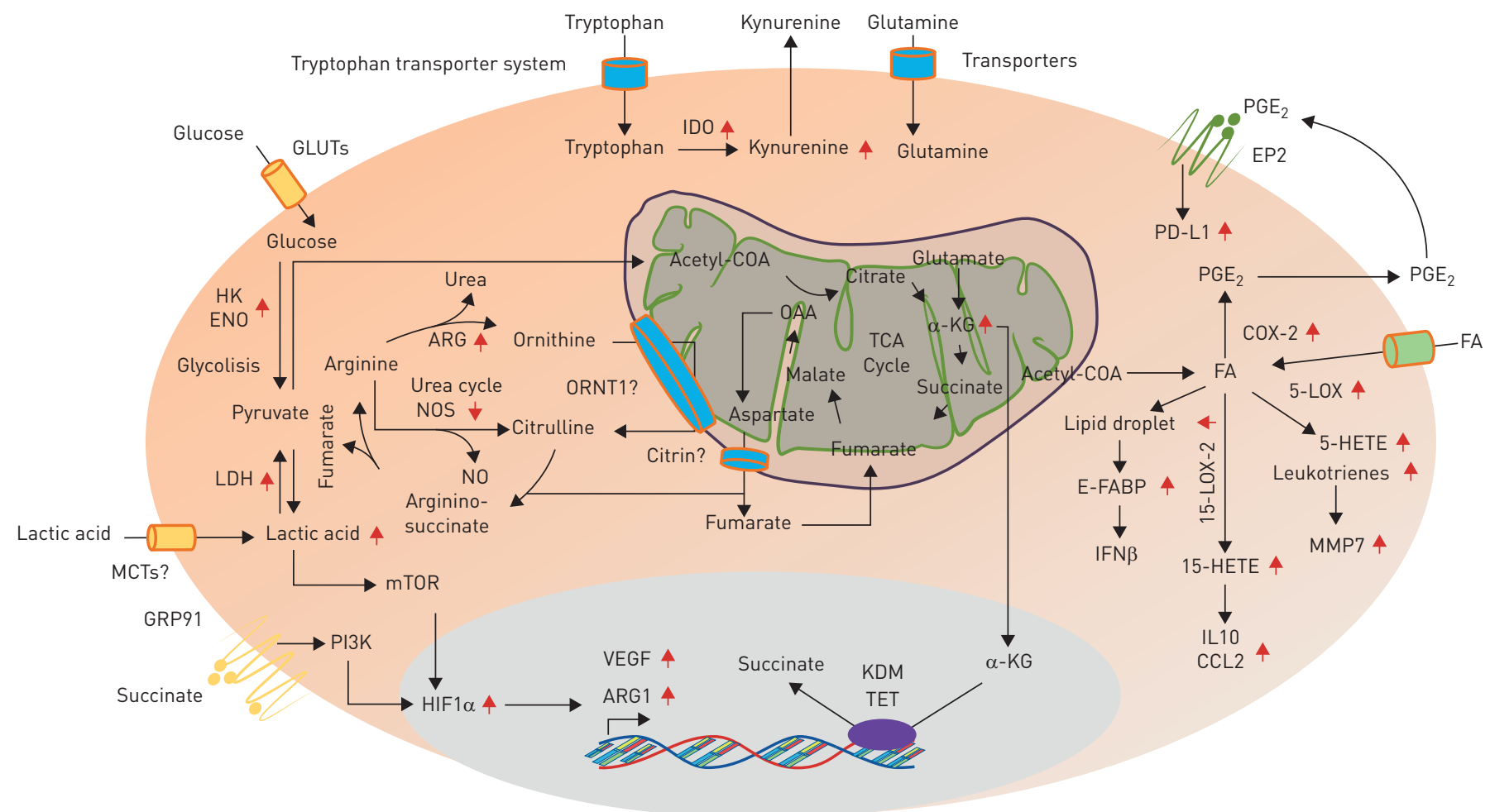

FIGURE 2 Metabolic architecture of tumour associated macrophages. Upward arrows indicate upregulation, downward arrows indicate downregulation and question marks illustrate the potential but inexplicit transporters. Transporters and/or metabolites of glucose metabolism are indicated as orange, amino acid metabolism as blue and lipid metabolism as green. IDO: indolamin-2,3-dioxygenase; GLUT: glucose transporter; HK: hexokinase; ENO: enolase; LDH: lactate dehydrogenase; MCTs: monocarboxylate transporters; ARG: arginase; NOS: nitric oxide synthase; NO: nitric oxide; mTOR: mammalian target of rapamycin; PI3K: phosphoinositide 3-kinases; HIF: hypoxia inducible factor; VEGF: vascular endothelial growth factor; KDM: histone lysine demethylase; TET: ten-eleven translocation; ORNT1: ornithine translocase; OAA: oxaloacetic acid; TCA cycle: tricarboxylic acid cycle; $\alpha$-KG: $\alpha$-ketoglutarate; COA: coenzyme $A_{;}$PGE 2 : prostaglandin $E_{2}$ EP2: prostaglandin E2 receptor 2; PD-L1: programmed death-ligand 1; FA: fatty acid; COX-2, cyclooxygenase-2; 5-LOX: 5-lipoxygenase; HETE: hydroxyeicosatetraenoic acid; MMP: matrix metalloproteinase; IL: interleukin; E-FABP: epidermal fatty acid binding proteins; 15-LOX-2: 15-lipoxygenase-2; IFN: interferon; GRP: G-protein-coupled receptor.

\section{Amino acid metabolism}

Amino acid metabolism by TAMs is a coadjutor of cancer cells for suppression of the T-cell immune response. The first evidence of this idea emerged four decades ago when it was shown that macrophages could suppress lymphocyte activity by arginine depletion in culture media [45]. Arginine is utilised by macrophages in either nitric oxide (NO) synthesis or in the arginase pathway to characterise M1- and M2-like polarisation, respectively [46, 47]. More precisely, M1 macrophages are characterised by production of inducible nitric oxide synthase (iNOS) to convert arginine into NO and L-citrulline, while M2 macrophages express arginase 1 (ARG1), which hydrolyses arginine to ornithine and urea. Protumoural TAMs express high levels of ARG1, thereby regulating immune evasion of Helicobacter pylori, which is one of the main causes of gastric cancer because it restrains macrophage iNOS expression and NO production and limits the generation of pro-inflammatory cytokines such as interferon (IFN) $-\gamma$, IL-17a and IL-12 [48]. Dysregulated metabolism of arginine by TAMs promotes tumour growth and development by impairing the antitumoural immune response. TAMs isolated from murine fibrosarcoma reveal immunosuppressive profiling with a low expression of iNOS [46]. In addition, macrophages overexpressing ARG1 demonstrate increased ARG1 activity with attenuated NO production, leading to a growth-promoting effect on breast cancer cells in vitro [47]. More importantly, high levels of ARG in TAMs reduce arginine in the TME, which is associated with loss of the $\zeta$-chain of CD3 in antigen-stimulated T-cells, thereby impairing T-cell antitumoural activity in lung cancer [49]. Interestingly, arginine availability can shift T-cell metabolism towards OXPHOS, rendering a higher survival capacity and antitumoural activity in activated T-cells [50]. Overall, it appears that TAMs, by depletion of arginine in the TME, not only suppress T-cell antitumoural activity, but also force T-cells to adopt glycolysis as their primary metabolic pathway. This is especially pronounced in hypoxic TMEs and in TMEs with high lactic acid levels, as TAMs show higher ARG expression; thus, arginine is reduced in the TME [51]. 
Tryptophan is another amino acid whose immunoregulatory role was discovered approximately two decades ago when researchers showed that IDO inhibition induced rapid T-cell-associated rejection of the allogeneic conceptus in mice [52]. A year later, the same group revealed that degradation of tryptophan by macrophage IDO suppressed T-cell proliferation in vitro, and the inhibition of IDO activity in macrophages reversed the anti-proliferative effect of macrophages on T-cells [53]. IDO is the first and rate-limiting enzyme in the kynurenine pathway, which converts tryptophan into $N$-formylkynurenine [54]. TAMs in different human tumour types, such as lung cancer, express high levels of IDO. The inhibitory effect of TAMs on T-cell proliferation and cytokine production (e.g. IFN- $\gamma$, TNF- $\alpha$ and IL-2) can be restored by adding exogenous tryptophan or inhibiting IDO [55]. Therefore, consumption of tryptophan in the TME by TAMs can deprive T-cells of tryptophan, which is necessary for biomass generation in activated T-cells, leading to accumulation of kynurenine as an immunosuppressive metabolite in the TME.

Glutamine is a well-known amino acid, which is particularly recognised for its role in the glutaminolysis pathway in cancer cells [56]. Glutamine synthase (GS) is the only enzyme in humans that is known to produce glutamine from glutamate. Interestingly, TAMs from human glioblastoma also exhibit upregulation of genes that are related to the transport and synthesis of glutamine, such as glutamateammonia ligase $(G L U L)$. Specific knockout of GS in macrophages reduces the metastatic rate in the LLC1 murine model. Although no significant change occurs in the TAM infiltration rate, GS knockout mice-derived TAMs are generally major histocompatibility complex class $\mathrm{II}^{\text {high }}$ and CD206 ${ }^{\text {low }}$ compared with their wild-type counterparts [57]. With regard to the TME, TAM-deficient GS enhances the level of intratumoural cytotoxic $\mathrm{CD}^{+}$T-cells. Moreover, GS knockout mice show increased vascular integrity, which was concomitant with a reduction in tumour hypoxia [57]. Overall, glutamine production by GS renders a protumoural phenotype in TAMs. However, glutamine and glutamate crosstalk between TAMs and cancer cells should be investigated more precisely because TAM-associated glutamine can be used to replenish the TCA cycle in cancer cells (figure 1) [58]. Furthermore, because glutamine uptake and metabolism is necessary for T-cell activation, competition between cancer cells and T-cells for TAM-associated glutamine indicates another level of cooperation for metabolites [59].

Amino acid-restricted TAMs exhibit an antitumoural phenotype with reduced TAM infiltration, tumour growth, and an increased response to immunotherapies in syngeneic tumour models of prostate and renal cell carcinoma (RCC) [34]. Hence, amino acid metabolism in TAMs exhibits a fundamental influence on phenotypic polarisation. Kynurenine is produced by tumour cells as a result of the enzymatic activity of IDO-1 and tryptophan 2,3-dioxygenase. Glioblastoma cell-derived kynurenine activates the aryl hydrocarbon receptor (AHR) in TAMs. By enhancing CCR2 expression, the AHR promotes TAM recruitment. Additionally, AHR drives protumoural polarisation of TAMs by upregulating Krüppel-like factor 4 and suppressing nuclear factor (NF)- $\mathrm{BB}$ activation [60]. Furthermore, glioblastoma cells secrete high levels of glutamate into the extracellular TME. TAMs isolated from human glioblastomas and co-cultured with glioblastoma cells display an immunosuppressive profile with upregulation of genes related to glutamate transport and metabolism, indicating that glioblastoma cell-derived glutamate might be utilised by TAMs to generate a protumoural phenotype [61]. The production of $\alpha$-ketoglutarate via glutaminolysis is important for M2 macrophage activation; however, whether intrinsic glutamate deprivation enhances the antitumoural phenotype of TAMs requires further investigation [62]. Moreover, hypoxia-induced semaphorin $3 \mathrm{~A}$ acts as an attractant for TAMs by triggering VEGF receptor 1 phosphorylation through neuropilin-1 (Nrp1) and plexin A1/plexin A4. Depletion of Nrp1 in TAMs is related to upregulation of iNOS and downregulation of ARG1 (figure 2) [63]. Therefore, hypoxia in the TME is one factor that enhances activation of the arginase pathway in TAMs. Researchers reported that TAM-derived IL-1 $\beta$ and TNF- $\alpha$ regulate arginine metabolism in neuroblastoma cells [64]; however, further studies are required to elucidate the cancer cell-derived triggers that affect arginine metabolism in TAMs.

\section{Lipid metabolism}

The lipogenic phenotype is one of the metabolic hallmarks of cancer. Fatty acid metabolism plays a pivotal role in regulating cancer cell survival and therapeutic resistance [65]. In addition to secreting leptin and transforming growth factor- $\beta$, which enhances cancer cell malignancy, adipocytes in the TME are a major lipid source [66]. Fatty acids are important substrates that are used in cellular membrane architecture, energy production and generation of signalling molecules. Additionally, lipid mediator products of fatty acids were reported to stimulate resolution in cancer by enhancing macrophage phagocytosis of tumour cell debris and counter-regulating the secretion of pro-inflammatory cytokines by macrophages [67].

Although glucose and amino acid metabolism mostly renders the protumoural phenotype of TAMs, metabolism of fatty acids in TAMs can act as a double-edged sword that can confer a protumoural function to TAMs and can lead to an antitumoural phenotype. Antitumoural TAMs express the epidermal 
fatty acid-binding protein (E-FABP), which is a lipid chaperone that can coordinate the distribution and metabolism of intracellular lipids. Upregulation of the E-FABP modulates the inflammatory response of TAMs, especially by increasing the activity of the IFN- $\beta$ signalling cascade. Given that the E-FABP is involved in lipid metabolism, inhibition of lipid droplet formation impairs IFN- $\beta$ production in macrophages, which illustrates an E-FABP-IFN- $\beta$ lipid droplet axis in protumoural TAM activation [68]. Furthermore, tumours from E-FABP ${ }^{-1-}$ mice contained a significantly higher percentage of $\mathrm{CD} 4^{+}, \mathrm{CD}^{+}$ T-cells, and natural killer cells in the early stages (e.g., 1 week after tumour implantation) [68]. Therefore, it seems that E-FABP-associated lipid metabolism of TAMs is involved in the antitumoural immune response, especially in the initial stages of tumour formation by arming TAMs with IFN- $\beta$ production and enhancement of immunoprotective cell recruitment to the tumour site.

Supporting the antitumoural function of lipid metabolism in TAMs, researchers recently showed that TAM extracellular vesicles (TAM-EVs) composed an immunostimulatory molecular pattern similar to antitumoural macrophages [69]. Lipid metabolism, especially the arachidonic acid pathway, is enriched in TAM-EVs. Notably, cyclooxygenase (COX)-1, thromboxane A synthase-1, and certain cytochrome P450 epoxygenases are upregulated in the proteome signature of TAM-EVs [69]. Interestingly, TAMs exert antitumoural functions by rewiring the arachidonic acid catabolic pathway in cancer cells through TAM-EVs, to induce $\mathrm{TXB}_{2}$ production and reduce prostaglandins [69]. This finding shows that lipid metabolism in the TME is fine-tuned by cellular crosstalk in which lipid metabolites exhibit intrinsic immune properties and can regulate the metabolic profile of other cell types. However, it is unknown how TAMs communicate with other immune cells through extracellular vesicles. Lipid metabolism also confers a protumoural phenotype to TAMs. RCC-derived TAMs produce a high amount of eicosanoid 15-hydroxyeicosatetraenoic acid (15-HETE), which is synthesised by 15-lipoxygenase (15-LOX). Conversely, the level of prostaglandin $\mathrm{E}_{2}\left(\mathrm{PGE}_{2}\right)$ was the same in RCC-derived TAMs and a normal kidney. RCC-derived TAMs show a higher expression of the 15-LOX2 isoform, concomitant with lower expression of COX-2, which is responsible for $\mathrm{PGE}_{2}$ production. Interestingly, inhibition of 15-LOX2 rather than COX-2 reduces IL-10 and CCL2 production by TAMs and consequently impairs the TAM protumoural phenotype [70]. The 5-LOX pathway has also been shown to be involved in lung cancer, with 5-LOX-expressing alveolar macrophages increased in the lungs of human hepatocellular carcinoma patients with lung metastasis by producing leukotriene $\mathrm{B}_{4}$, a potent tumour growth promoting mediator [71]. In a mouse model, deactivating myelocytomatosis (MYC) lowered 5-LOX mRNA levels and inhibiting 5-LOX in vivo reduced leukotriene $\mathrm{B}_{4}$ levels as well as lung tumour burden [72]. The extracellular vesicles from lung cancer pleural exudates were shown to transform cysteinyl-leukotriene $\mathrm{C}_{4}$ $\left(\mathrm{LTC}_{4}\right)$ to $\mathrm{LTD}_{4}$, which stimulated cancer cell migration and survival [73]. Accordingly, the pro-metastatic effect of exosomes can be mediated by the leukotriene machinery, further supporting the use of CysLT1 receptor antagonists for lung cancer therapy (e.g. montelukast). Additionally, inhibition of lipid droplet formation in vitro can reduce the antitumoural capability of TAMs [68]. Furthermore, targeting lipid droplet formation in TAMs through reduction of fatty acid transportation into lipid droplets impairs tumour growth in murine models by decreasing the proportion of protumoural TAMs in tumours [74]. Recently, it has been shown that TAMs increase the lipid accumulation via upregulation of scavenger receptor CD36. TAMs use the accumulated lipids as a source of energy by fatty acid oxidation. Interestingly, inhibition of lipid uptake by blocking CD36 or suppression of fatty acid oxidation in macrophages inhibit the generation of TAMs, thereby reduce the protumour functions of TAMs [75]. Therefore, depending on the tumour stage, TAMs can use lipid metabolism as either a protumoural or an antitumoural tool. Moreover, the fate of lipid precursors can differ based on the phenotype and function of TAMs.

Increased intracellular enrichment of lipids is associated with infiltration and phenotypic switching in TAMs. The effects depend on multiple factors, including lipid content and cancer types and stages. For example, TAMs enriched with the polyunsaturated fatty acid (PUFA) and linoleic acid (18:2) demonstrate antitumoural effects on early stage breast tumours and protumoural effects on human ovarian carcinoma, while TAMs accumulated with the saturated fatty acid, stearic acid (18:0), or the PUFA arachidonic acid (20:4) primarily display protumoural functions [68, 70,76-80]. In response to linoleic acid treatment, the E-FABP, an intracellular lipid chaperone that is highly expressed in M1 macrophages, was upregulated in TAMs to enhance IFN- $\beta$ responses by increasing lipid droplet formation in the early stages (e.g., 1 week after tumour implantation) in a murine breast tumour model [68]. Stearic acid did not exert a similar M1 polarisation effect, which is in agreement with the finding that macrophages enriched with stearic acid are not tumouricidal $[68,80]$. In contrast, TAMs accumulated in the later stages (on average 3 weeks after tumour implantation) of breast cancer exhibited high adipocyte/macrophage FABP expression, which promoted tumour growth via IL-6/STAT3 signalling [81]. Lipid droplet formation in the late stages of murine breast tumour models correlates with TAM protumoural polarisation; caspase-1 inactivates medium-chain acyl-CoA dehydrogenase by cleaving peroxisome proliferator-activated receptor (PPAR)- $\gamma$ 
at Asp64, thereby inhibiting fatty acid oxidation and inducing lipid droplet formation in TAMs [82]. However, it is unclear if adipocyte FABP expression in advanced tumour stages occurs in a lipid droplet formation-dependent manner. Moreover, high concentrations of linoleic acid and arachidonic acid in the TME are agonists of PPAR $\beta / \delta$ and facilitate protumoural polarisation of TAMs $[82,83]$. Lipid droplet formation serves as a pool to enrich TAMs with PPAR $\beta / \delta$ ligands, leading to upregulation of PPAR $\beta / \delta$ target genes and polarisation of TAMs to a protumoural phenotype in ovarian carcinoma [76]. In addition to cancer cells, immune cells can also shape the metabolic profile of TAMs. Recently, it has been shown that Tregs can promote protumoural TAMs by modulating lipid metabolism. Mechanistically, Tregs block the inhibitory effect of $\mathrm{CD}^{+}$T-cell-associated IFN- $\gamma$ on sterol regulatory element-binding protein- 1 of TAMs, inducing fatty acid synthesis in M2-like TAMs [84].

Increased arachidonic acid metabolism is associated with protumoural polarisation of TAMs. Arachidonic acid can be utilised to synthesise $\mathrm{PGE}_{2}$ through the activated phospholipase $\mathrm{A}_{2} / \mathrm{COX}-2 /$ microsomal $\mathrm{PE}_{2}$ synthase 1 (mPGES1) pathway. PDAC-derived arachidonic acid is delivered to TAMs via exosomes, leading to an immunosuppressive protumoural phenotype with increased secretion of protumoural molecules such as PGE $_{2}$, VEGF, monocyte chemoattractant protein-1, IL-6 and matrix metallopeptidase (MMP)-9 [77]. In addition, tumour cells can induce expression of programmed cell death protein ligand 1 (PD-L1) in TAMs via the COX-2/mPGES1/PGE 2 pathway, which deactivates cytotoxic T-cells and facilitates tumour escape from immune surveillance in murine bladder tumours [78]. Aside from the effect on polarisation of TAMs, arachidonic acid metabolism influences TAM infiltration in the TME. The enzyme 5-LOX converts arachidonic acid into 5-HETE and leukotrienes. Under hypoxic conditions, ovarian cancer cells produce high levels of 5-LOX to promote TAM infiltration, through upregulation of MMP-7 [79]. Similarly, the 15-LOX-2 pathway in TAMs is activated in human RCC, resulting in an increase in the secretion of the arachidonic acid metabolite 15-HETE, the chemokine CCL2, and the immunosuppressive cytokine IL-10 [71]. Monocytes are recruited to the site by binding to CCR2, while IL-10 mediates the development of immune tolerance $[23,70]$. This suggests that arachidonic acid directly influences TAM infiltration and polarisation in the TME (figure 2).

\section{Metabolic reprogramming of TAMs to favour a tumouricidal phenotype as a potential antitumoural therapeutic strategy}

Specialised tumour metabolism supports cancer cell energetics and plays a critical role in establishing an immunosuppressive TME. Blocking TAM recruitment is one of the approaches that can be used to inhibit tumour progression [23]. 5-LOX metabolites facilitate TAM infiltration, but blocking recruitment of TAMs by manipulating 5-LOX should be further investigated [79]. Nevertheless, repolarising TAMs towards an antitumoural phenotype is more effective than blocking recruitment to prevent tumour growth [85]. $\beta$-catenin plays a pivotal role as a regulatory hub in several cellular processes including metabolism [86]. A recent study from our group provides strong evidence that $\beta$-catenin-mediated transcription plays a central role in the transition of tumour-inhibiting M1-like TAMs to tumour-promoting M2-like TAMs. Therefore, targeting $\beta$-catenin in TAMs may provide a new immunotherapeutic option to reactivate antitumour immunity in the TME of the lung [87].

Activated caspase-1, which causes lipid accumulation and differentiation of TAMs towards a protumoural phenotype, is exclusively detectable in TAMs but not in tumour cells or normal tissues. Hence, caspase-1 inhibition is a potential strategy for reprograming the TME. Caspase-1 inhibitors, including NCX-4016, YVAD and VAD, repolarise TAMs towards an antitumoural phenotype and suppress tumour growth in vivo [82]. Given that the COX-2/mPGES1/PGE 2 pathway is involved in the regulation of $\mathrm{PD}-\mathrm{L} 1$ expression in TAMs, selective inhibition of COX-2, mPGES1 or genetic overexpression of the $\mathrm{PGE}_{2}$-degrading enzyme, 15-hydroxyprostaglandin dehydrogenase, could alleviate immune suppression and re-establish the antitumour immune response in the TME $[78,88]$. Cancer cells promote TAM membrane cholesterol efflux, which is regulated by the ATP-binding cassette transporter (ABC transporter), leading to protumoural programming with inhibition of IFN- $\gamma$-induced gene expression in TAMs. Genetic deletion of $\mathrm{ABC}$ transporters reverts the tumour-promoting functions of TAMs to an antitumoural phenotype in murine bladder carcinoma and melanoma, as well as in ovarian carcinoma models [89, 90]. Overexpression of $\mathrm{ABC}$ transporters confers multidrug resistance through increasing efflux of drugs from cancer cells. However, clinical trials demonstrated that application of $\mathrm{ABC}$ transporter inhibitors as chemotherapeutic sensitisers exhibits limited or no benefit for patients with cancer [91]. Additionally, studies suggest that preventing cholesterol efflux in TAMs by targeting the ABC transporter could block the protumoural functions of TAMs to restore antitumoural immunity $[89,90]$. Therefore, the development of TAM-specific $\mathrm{ABC}$ transporter inhibition strategies might be a potential novel antitumoural therapeutic approach.

Aerobic glycolysis is a hallmark of cancer cells. Tumour cell-derived lactic acid drives TAMs to favour a protumoural state $[40,92]$. Nonspecific inhibition of the glycolytic pathway, which involves 
downregulation of pyruvate kinase isozyme 2 as a promoter of aerobic glycolysis, in a CT26 colon cancer cell-M2 macrophage co-culture system reverses macrophage M2 polarisation by reducing CD206 and legumain, while inducing STAT1 and TNF- $\alpha$ [93]. Additionally, administration of the pyruvate dehydrogenase kinase inhibitor, dichloroacetate, enhances the tumouricidal activity of TAMs in a murine T-cell lymphoma model. TAMs are one of the major producers of IL-1 $\beta$, which promotes tumour progression. Production of IL- $1 \beta$ by TAM activation of both the NF- $\kappa B$ and $m T O R$ pathways occurs in a glucose-dependent manner [94]. A prototypical inhibitor of $\mathrm{mTOR}$, rapamycin, repolarises protumoural macrophages towards an antitumoural phenotype by suppressing mitochondrial ROS and NLRP3 inflammasomes, suggesting that targeting upper stream factor of glucose would be beneficial for antitumoural responses in TAMs $[95,96]$. Expectedly, the glucose-lowering drug, metformin, reduces M2 polarisation in TAMs in murine pancreatic tumours and osteosarcoma tumour models and reduces IL-1 $\beta$ production [97, 98]. Similarly, inhibition of glucose metabolism in the TME with 2-deoxyglucose, a competitive inhibitor of HK2, impairs IL-1 $\beta$ production [94]. Moreover, instead of inhibiting the glycolytic pathway in the entire TME, specific inhibition of aerobic glycolysis with 2-deoxyglucose in protumoural TAMs is already sufficient to disrupt the prometastatic phenotype of TAMs [99].

Protumoural TAMs are characterised by upregulation of ARG1, which is promoted by the Ron-receptor tyrosine kinase (RTK), which is specifically expressed by protumoural TAMs [34]. This suggests that targeting RTK activity using RTK inhibitors such as sunitinib malate in protumoural TAMs could be therapeutically beneficial. Additionally, specific targeting of ARG in TAMs could exhibit significant therapeutic implications. The ARG1 inhibitor, L-norvaline, reverses the proliferative effect of protumoural TAMs overexpressed with ARG1 in vitro [47]. Moreover, anti-PD-1 therapy decreases the expression of ARG1 in TAMs, which is in line with the principle of PD-1 targeting immunotherapy [100]. Given that the rate-limiting enzyme for arginine biosynthesis, arginine-succinate synthetase (ASS1), may be elevated in TAMs, further research should be carried out to investigate the role of ASS1 in channelling arginine to ARG and iNOS (table 1) [101].

\section{Future perspectives}

One of the main challenges in targeted cell therapy is finding the molecular pathways, which are specific and unique to the interested cell. This challenge has a higher level of complexity, especially for TAMs by considering their plasticity. Studies on immunometabolism, in particular the metabolism of TAMs, provide novel therapeutic opportunities to TAMs-targeted therapy. In this line, finding metabolism-associated functions in TAMs can open new avenues for TAM-based therapy. Recruitment and migration of TAMs are two energy-consuming pathways. Although it has been shown that glycolysis impacts macrophage migration [22], the involvement of other metabolic pathways especially, OXPHOS and fatty acid oxidation in actin and cytoskeleton rearrangement, as two main migration compartments, need to be investigated during monocyte recruitment into the tumour site [22]. Furthermore, based on significant roles of metabolism in trained immunity, deciphering the metabolism contribution to trained immunity of TAMs can reveal novel specific targets involving TAMs plasticity and epigenetic programming [111]. In regard to the tumour acidosis that is dependent upon metabolic status of TME cellular components [112], it has been shown that TME acidification can induce pro-tumour TAMs whereas the contribution of TAMs in TME acidosis is completely unknown [113]. As modulation of TME acidosis can improve the immunotherapy [114], deciphering the TAMs-associated acidosis can reveal some novel opportunities to use not only for specific targeting of TAMs, but also for combination therapy especially in the immune checkpoint field. TAMs display a high level of functional plasticity that is symbolised by high sensitivity to the surrounding TME for phenotypic alteration. Therefore, aside from interference with TAM survival and inhibition of TAM infiltration, repolarisation of protumoural TAMs towards an antitumoural phenotype is a potential therapeutic approach for cancer.

Several strategies for reprogramming protumoural TAMs exist, such as manipulation of TME stimuli and influencing the NF- $\mathrm{BB}$, mitogen-activated protein kinase/extracellular signal-regulated kinase, and Wnt/ $B$-catenin pathways $[23,87]$. Although evidence shows that metabolic regulation is crucial to phenotypic switching in TAMs, such as metabolic regulation by metformin [97, 98], better characterisation of the mechanisms underlying the intrinsic metabolic signals that drive TAM activation via cross-talk with cancer cell-derived metabolites is required.

As Treg cells can regulate TAMs metabolism, our surmise is other TME cellular components also can modulate TAMs metabolism [84]. However, further studies are needed to identify the immune/stromal cells metabolism cross-talk with TAMs. In addition, epigenetic regulation, which is strongly affected by metabolic changes, plays a pivotal role in macrophage polarisation. For instance, $\alpha$-ketoglutarate is important for regulating Jmjd3-dependent polarisation in M2 macrophages [62]. A need exists for more preclinical studies to identify the epigenetic and metabolic networks that reprogram protumoural TAMs as a means to enhance cancer immunotherapy. 
TABLE 1 Selected agents targeting metabolism for treatment of various metabolic or inflammatory diseases and cancer

\begin{tabular}{|c|c|c|c|c|c|}
\hline Drug & Target & Metabolic pathway/consequence & Disease model & Clinical trials/status & Effect on TAMs \\
\hline \multicolumn{6}{|l|}{ Glucose metabolism } \\
\hline 2-deoxyglucose (2-DG) \# & $\begin{array}{l}\text { Hexokinase } 2 \\
\quad \text { (glucose uptake) }\end{array}$ & $\begin{array}{l}\text { Inhibition of glucose uptake and } \\
\text { therefore aerobic glycolysis }\end{array}$ & $\begin{array}{l}\text { Cancer in general, rheumatoid } \\
\text { arthritis }\end{array}$ & $\begin{array}{l}\text { Stopped due to toxicity } \\
\quad \text { (hypoglycaemia) [102] }\end{array}$ & Repolarisation [34] \\
\hline $\begin{array}{l}\text { Enasidenib (AG-221), } \\
\text { AG-120 (Ivosidenib), } \\
\text { AGI-5198, AG-881, }\end{array}$ & Mutant IDH1/2 & $\begin{array}{l}\text { Inhibition of } \alpha-K G \text { reduction to } 2-H G \text { by } \\
\text { mutant IDH leading to impaired } \\
\text { demethylation }\end{array}$ & $\begin{array}{l}\text { Acute myeloid leukaemia, bile duct } \\
\text { cancer, glioma, haematological } \\
\text { malignancies, solid tumours } \\
{[103,104]}\end{array}$ & $\begin{array}{l}\text { Enasidenib and ivosidenib } \\
\text { approved for acute } \\
\text { myeloid leukaemia }\end{array}$ & \\
\hline CB-839 & Glutaminase 1 & $\begin{array}{l}\text { Inhibition of glutamin metabolism } \\
\text { (increased dependence of glutamine } \\
\text { in cancer cells) }[105,106]\end{array}$ & $\begin{array}{l}\text { Colorectal cancer, NSCLC, renal cell } \\
\text { carcinoma, melanoma }\end{array}$ & $\begin{array}{l}\text { NCT03263429, } \\
\text { NCT03831932, } \\
\text { NCT02771626 }\end{array}$ & \\
\hline Metformin ${ }^{\#}$ & AMPK & $\begin{array}{l}\text { Reduction in glycolytic pathway, } \\
\text { reduced glucose blood levels, } \\
\text { increased FAO, inhibition of } \\
\text { respiration, inhibition of mTOR }\end{array}$ & $\begin{array}{l}\text { Type II diabetes, cancer in general, } \\
\text { rheumatoid arthritis }\end{array}$ & $\begin{array}{l}\text { Approved for type II } \\
\text { diabetes, } \\
\text { NCT02019979, } \\
\text { NCT02640534, } \\
\text { NCT01310231, } \\
\text { NCT02312661 }\end{array}$ & $\begin{array}{l}\text { Repolarisation } \\
{[97,98]}\end{array}$ \\
\hline \multicolumn{6}{|l|}{ Nucleotide biosynthesis } \\
\hline $\begin{array}{l}\text { Methotrexate, } \\
\text { Pemetrexed }\end{array}$ & DHFR, GARFT & Impaired nucleotide biosynthesis & Breast cancer & $\begin{array}{l}\text { Phase II trial } \\
\text { (methodextrate) }\end{array}$ & \\
\hline \multicolumn{6}{|l|}{ Amino acid metabolism } \\
\hline $\begin{array}{l}\text { L-asparaginase (Elspar, } \\
\text { Kidrolase), } \\
\text { PEG-BCT-100 } \\
\text { (ADI-PEG20), } \\
\text { AEB-1102 }\end{array}$ & Circulating arginine & $\begin{array}{l}\text { Breakdown of arginin, targeting cancer } \\
\text { cells without ability for arginase de } \\
\text { novo synthesis (ASS1 silenced cancer } \\
\text { types) }\end{array}$ & $\begin{array}{l}\text { Melanoma, hepatocellular carcinoma, } \\
\text { acute lymphocytic leukaemia }\end{array}$ & $\begin{array}{l}\text { L-asparaginase approved for } \\
\text { acute lymphocytic } \\
\text { leukaemia }\end{array}$ & \\
\hline Rapamycin, RAD001 ${ }^{\#}$ & mTOR & $\begin{array}{l}\text { Deregulation of proliferation and } \\
\text { protein/lipid/nucleotide production }\end{array}$ & ALS, glioma, NSCLC & $\begin{array}{l}\text { NCT03359538, } \\
\text { NCT01158651, } \\
\text { NCT01063478 }\end{array}$ & $\begin{array}{l}\text { Repolarisation } \\
{[95,96]}\end{array}$ \\
\hline L-norvaline, CB-1158\# & Arginase 1 & Disruption of de novo arginine synthesis & $\begin{array}{l}\text { Advanced solid tumours, Alzheimer's } \\
\text { disease models [107] }\end{array}$ & & Repolarisation [47] \\
\hline PHGDH inhibitors & $\begin{array}{c}\text { Phosphoglycerate } \\
\text { dehydrogenase }\end{array}$ & De novo serine synthesis & $\begin{array}{l}\text { Breast cancer, lung adenocarcinoma, } \\
\text { melanoma }[108-110]\end{array}$ & & \\
\hline \multicolumn{6}{|l|}{ Lipid metabolism } \\
\hline ND-646 & $\mathrm{ACC}$ & Impaired de novo fatty acid synthesis & Lung tumour models [82] & & \\
\hline $\begin{array}{l}\text { Pralnacasan, NCX-4016, } \\
\text { YVAD, VAD\# }\end{array}$ & Caspase-1 & $\begin{array}{l}\text { Inhibition of inflammasome/lipid } \\
\text { accumulation in inflammatory cells }\end{array}$ & $\begin{array}{l}\text { Rheumatoid arthritis, osteoarthritis, } \\
\text { inflammatory bowel disorders, } \\
\text { cancer, autoimmune diseases }\end{array}$ & $\begin{array}{l}\text { Pralnacasan studies stopped } \\
\text { after phase II }\end{array}$ & $\begin{array}{l}\text { Specifically targets } \\
\text { and repolarises } \\
\text { TAMs [82] }\end{array}$ \\
\hline $\begin{array}{l}\text { Paclitaxel, } \\
\text { Methodextrate, } \\
\text { Doxorubicin\# }\end{array}$ & $A B C$ transporter & $\begin{array}{l}\text { Impaired efflux leading to accumulation } \\
\text { of e.g. xenobiotics or cholesterol in } \\
\text { the cell }\end{array}$ & Multidrug resistant cancer [91] & $\begin{array}{l}\text { Studies ongoing e.g. phase } \\
\text { III trial for breast cancer } \\
\text { (NCT02488967) }\end{array}$ & Repolarisation [90] \\
\hline $\begin{array}{l}\text { Meclofenamate sodium, } \\
\text { Zileuton\# }\end{array}$ & 5-LOX & $\begin{array}{l}\text { Conversion of arachidonic acid to } \\
5 \text {-HETE and leukotrienes }\end{array}$ & $\begin{array}{l}\text { Pain relief, rheumatoid arthritis, } \\
\text { osteoarthritis, asthma }\end{array}$ & & TME infiltration [79] \\
\hline
\end{tabular}

TAM: tumour-associated macrophages; IDH: isocitrate dehydrogenase; $\alpha$-KG: $\alpha$-ketoglutarate; 2 -HG: 2-hydroxyglutaric acid; NSCLC: nonsmall cell lung cancer; AMPK: AMP-activated protein kinase; FAO: fatty acid oxidation; mTOR: mammalian target of rapamycin; DHFR: dihydrofolate reductase; GARFT: glycinamide ribonucleotide formyltransferase; ALS: amyotrophic lateral sclerosis; ACC: acetyl-CoA carboxylase; TME: tumour microenvironment; 5-LOX: 5-lipoxygenase; 5-HETE: 5-hydroxyeicosatetraenoic acid. "\#: potential specific TAM metabolic targets. 
Selective targeting of TAM metabolism in vivo is an ongoing challenge. As mentioned previously, cichloroacetate exhibits potential in TAM M2-to-M1 reprogramming. However, dichloroacetate also inhibits aerobic glycolysis and induces differentiation of Tregs, which might result in decreased immunosurveillance in cancer therapy [115]. Hence, the therapeutic potential of dichloroacetate might be hampered in the absence of a specific TAM-targeting strategy. Although nanoparticle and liposome-based systems aid in efficient drug delivery to TAMs, the dynamic ability of TAMs to adapt to a specific microenvironment increases the difficulty for in vivo metabolic targeting. Therefore, investigations into the metabolic features of TAMs at a spatial and temporal resolution using specialised experimental technologies, such as in vivo tracer analysis and single-cell technologies in combination with high-resolution mass spectrometry for accurate metabolite identification, would offer more precise guidance for metabolic regulation [116, 117]. Furthermore, depletion of TAMs generates a less hypoxic TME and reduces tumour glycolysis, leading to increased PD-L1 expression in tumours. As some patients with lung cancer acquire resistance to immune checkpoint therapies and some groups do not respond [118], a combination of immunotherapeutic agents such as PD-L1 inhibitors and TAM metabolic interventions could be beneficial for cancer therapy.

Conflict of interest: None declared.

Support statement: This work was supported by the Max Planck Society, Verein zur Förderung der Krebsforschung in Gießen e.V., Von-Behring-Röntgen-Stiftung, a Rhön Klinikum AG grant, Institute for Lung Health (ILH), Cardio-Pulmonary Institute (CPI), the German Center for Lung Research (DZL). DFG, SFB CRC 1213 (Project A01, A05 to Pullamsetti SS and project A10* to Savai R), European Research Council (ERC) Consolidator Grant (\#866051 to Pullamsetti SS) and Frankfurt Cancer Institute (LOEWE FCI).

\section{References}

Barta JA, Powell CA, Wisnivesky JP. Global epidemiology of lung cancer. Ann Glob Health 2019; 85: 8. Siegel RL, Miller KD, Jemal A. Cancer statistics, 2020. CA Cancer J Clin 2020; 70: 7-30.

Altorki NK, Markowitz GJ, Gao D, et al. The lung microenvironment: an important regulator of tumour growth and metastasis. Nat Rev Cancer 2019; 19: 9-31.

4 El-Nikhely N, Larzabal L, Seeger W, et al. Tumor-stromal interactions in lung cancer: novel candidate targets for therapeutic intervention. Expert Opin Investig Drugs 2012; 21: 1107-1122.

5 Sarode P, Schafer MB, Grimminger F, et al. Macrophage and tumor cell cross-talk is fundamental for lung tumor progression: we need to talk. Front Oncol 2020; 11: 324.

6 Bach LA, Sumpter DJT, Alsner J, et al. Spatial evolutionary games of interaction among generic cancer cells. J Theoretical Med 2003; 5: 47-58.

7 Bach LA, Bentzen SM, Alsner J, et al. An evolutionary-game model of tumour-cell interactions: possible relevance to gene therapy. Eur J Cancer 2001; 37: 2116-2120.

8 Schmall A, Al-Tamari HM, Herold S, et al. Macrophage and cancer cell cross-talk via CCR2 and CX3CR1 is a fundamental mechanism driving lung cancer. Am J Resp Crit Care 2015; 191: 437-447.

9 Priceman SJ, Sung JL, Shaposhnik Z, et al. Targeting distinct tumor-infiltrating myeloid cells by inhibiting CSF-1 receptor: combating tumor evasion of antiangiogenic therapy. Blood 2010; 115: 1461-1471.

10 Lohela $\mathrm{M}$, Casbon AJ, Olow A, et al. Intravital imaging reveals distinct responses of depleting dynamic tumor-associated macrophage and dendritic cell subpopulations. Proc Natl Acad Sci USA 2014; 111: E5086-E5095.

11 Zhu Y, Knolhoff BL, Meyer MA, et al. CSF1/CSF1R blockade reprograms tumor-infiltrating macrophages and improves response to T-cell checkpoint immunotherapy in pancreatic cancer models. Cancer Res 2014; 74: 5057-5069.

12 Salazar Y, Zheng X, Brunn D, et al. Microenvironmental Th9- and Th17- lymphocytes induce metastatic spreading in lung cancer. J Clin Invest 2020; 130: 3560-3575.

13 Dumitriu IE, Dunbar DR, Howie SE, et al. Human dendritic cells produce TGF-beta 1 under the influence of lung carcinoma cells and prime the differentiation of CD4+CD25+Foxp3+ regulatory T cells. J Immunol 2009; 182: 2795-2807.

14 Coffelt SB, Wellenstein MD, de Visser KE. Neutrophils in cancer: neutral no more. Nat Rev Cancer 2016; 16: 431-446.

15 Wang TT, Liu GW, Wang RN. The intercellular metabolic interplay between tumor and immune cells. Front Immunol 2014; 5: 358.

16 Gupta S, Roy A, Dwarakanath BS. Metabolic cooperation and competition in the tumor microenvironment: implications for therapy. Front Oncol 2017; 7: 68.

17 Lewis CE, Pollard JW. Distinct role of macrophages in different tumor microenvironments. Cancer Res 2006; 66: 605-612.

18 Yahaya MAF, Lila MAM, Ismail S, et al. Tumour-associated macrophages (TAMs) in colon cancer and how to reeducate them. J Immunol Res 2019; 2019: 2368249.

19 Chanmee $\mathrm{T}$, Ontong $\mathrm{P}$, Konno $\mathrm{K}$, et al. Tumor-associated macrophages as major players in the tumor microenvironment. Cancers (Basel) 2014; 6: 1670-1690.

20 Cassetta L, Kitamura T. Targeting tumor-associated macrophages as a potential strategy to enhance the response to immune checkpoint inhibitors. Front Cell Dev Biol 2018; 6: 38.

21 Qiu SQ, Waaijer SJH, Zwager MC, et al. Tumor-associated macrophages in breast cancer: Innocent bystander or important player? Cancer Treat Rev 2018; 70: 178-189.

22 Tan SY, Krasnow MA. Developmental origin of lung macrophage diversity. Development 2016; 143: 1318-1327. 
Zheng X, Turkowski K, Mora J, et al. Redirecting tumor-associated macrophages to become tumoricidal effectors as a novel strategy for cancer therapy. Oncotarget 2017; 8: 48436-48452.

Netea-Maier RT, Smit JWA, Netea MG. Metabolic changes in tumor cells and tumor-associated macrophages: a mutual relationship. Cancer Lett 2018; 413: 102-109.

De Palma M, Biziato D, Petrova TV. Microenvironmental regulation of tumour angiogenesis. Nat Rev Cancer 2017; 17: 457-474.

Leach RM, Treacher DF. Oxygen transport-2. Tissue hypoxia. BMJ 1998; 317: 1370-1373.

Semba H, Takeda N, Isagawa T, et al. HIF-1alpha-PDK1 axis-induced active glycolysis plays an essential role in macrophage migratory capacity. Nat Commun 2016; 7: 11635.

Pantaloni D, Le Clainche C, Carlier MF. Mechanism of actin-based motility. Science 2001; 292: 1502-1506.

Gocheva V, Wang HW, Gadea BB, et al. IL-4 induces cathepsin protease activity in tumor-associated macrophages to promote cancer growth and invasion. Genes Dev 2010; 24: 241-255.

Lin EY, Gouon-Evans V, Nguyen AV, et al. The macrophage growth factor CSF-1 in mammary gland development and tumor progression. J Mammary Gland Biol Neoplasia 2002; 7: 147-162.

Liu D, Chang C, Lu N, et al. Comprehensive proteomics analysis reveals metabolic reprogramming of tumor-associated macrophages stimulated by the tumor microenvironment. J Proteome Res 2017; 16: 288-297.

Vitale I, Manic G, Coussens LM, et al. Macrophages and metabolism in the tumor microenvironment. Cell Metab 2019; 30: 36-50.

Arts RJW, Plantinga TS, Tuit S, et al. Transcriptional and metabolic reprogramming induce an inflammatory phenotype in non-medullary thyroid carcinoma-induced macrophages. Oncoimmunology 2016; 5: e1229725.

Penny HL, Sieow JL, Adriani G, et al. Warburg metabolism in tumor-conditioned macrophages promotes metastasis in human pancreatic ductal adenocarcinoma. Oncoimmunology 2016; 5: e1191731.

Henze AT, Mazzone M. The impact of hypoxia on tumor-associated macrophages. J Clin Invest 2016; 126: 3672-3679.

Wenes M, Shang M, Di Matteo M, et al. Macrophage metabolism controls tumor blood vessel morphogenesis and metastasis. Cell Metab 2016; 24: 701-715.

Jeong H, Kim S, Hong BJ, et al. Tumor-associated macrophages enhance tumor hypoxia and aerobic glycolysis Cancer Res 2019; 79: 795-806.

Herzig S, Shaw RJ. AMPK: guardian of metabolism and mitochondrial homeostasis. Nat Rev Mol Cell Bio 2018 ; 19: 121-135.

Gatenby RA, Gillies RJ. Why do cancers have high aerobic glycolysis? Nat Rev Cancer 2004; 4: 891-899.

Colegio OR, Chu NQ, Szabo AL, et al. Functional polarization of tumour-associated macrophages by tumour-derived lactic acid. Nature 2014; 513: 559-563.

Liu N, Luo J, Kuang D, et al. Lactate inhibits ATP6V0d2 expression in tumor-associated macrophages to promote HIF-2 alpha-mediated tumor progression. J Clin Invest 2019; 129: 631-646.

Shime H, Yabu M, Akazawa T, et al. Tumor-secreted lactic acid promotes IL-23/IL-17 proinflammatory pathway. Immunol 2008; 180: 7175-7183.

El-Kenawi A, Gatenbee C, Robertson-Tessi M, et al. Acidity promotes tumour progression by altering macrophage phenotype in prostate cancer. Br J Cancer 2019; 121: 556-566.

Wu JY, Huang TW, Hsieh YT, et al. Cancer-derived succinate promotes macrophage polarization and cancer metastasis via succinate receptor. Mol Cell 2020; 77: 213-227 e215.

Kung JT, Brooks SB, Jakway JP, et al. Suppression of in vitro cytotoxic response by macrophages due to induced arginase. J Exp Med 1977; 146: 665-672.

Biswas SK, Gangi L, Paul S, et al. A distinct and unique transcriptional program expressed by tumor-associated macrophages (defective NF-kappa B and enhanced IRF-3/STAT1 activation). Blood 2006; 107: $2112-2122$.

Chang CI, Liao JC, Kuo L. Macrophage arginase promotes tumor cell growth and suppresses nitric oxide-mediated tumor cytotoxicity. Cancer Res 2001; 61: 1100-1106.

Lewis ND, Asim M, Barry DP, et al. Immune evasion by Helicobacter pylori is mediated by induction of macrophage arginase II. J Immunol 2011; 186: 3632-3641.

Rodriguez PC, Quiceno DG, Zabaleta J, et al. Arginase I production in the tumor microenvironment by mature myeloid cells inhibits T-cell receptor expression and antigen-specific T-cell responses. Cancer Res 2004; 64: 5839-5849.

Geiger R, Rieckmann JC, Wolf $\mathrm{T}$, et al. L-arginine modulates $\mathrm{T}$ cell metabolism and enhances survival and anti-tumor activity. Cell 2016; 167: 829-842 e813.

Carmona-Fontaine C, Deforet M, Akkari L, et al. Metabolic origins of spatial organization in the tumor microenvironment. Proc Natl Acad Sci USA 2017; 114: 2934-2939. Munn DH, Zhou M, Munn DH, Shafizadeh E, Attwood JT, et al. Inhibition of T cell proliferation by macrophage tryptophan catabolism. J Exp Med 1999; 189: 1363-1372.

Moffett JR, Namboodiri MA. Tryptophan and the immune response. Immunol Cell Biol 2003; 81: 247-265.

Zhao Q, Kuang DM, Wu Y, et al. Activated CD69+ T cells foster immune privilege by regulating IDO expression in tumor-associated macrophages. J Immunol 2012; 188: 1117-1124.

Yang L, Venneti S, Nagrath D. Glutaminolysis: a hallmark of cancer metabolism. Annu Rev Biomed Eng 2017; 19: 163-194.

Palmieri EM, Menga A, Martin-Perez R, et al. Pharmacologic or genetic targeting of glutamine synthetase skews macrophages toward an M1-like phenotype and inhibits tumor metastasis. Cell Rep 2017; 20: 1654-1666. Mazzone M, Menga A, Castegna A. Metabolism and TAM functions-it takes two to tango. FEBS J 2018; 285: $700-716$.

Carr EL, Kelman A, Wu GS, et al. Glutamine uptake and metabolism are coordinately regulated by ERK/MAPK during T lymphocyte activation. J Immunol 2010; 185: 1037-1044.

Takenaka MC, Gabriely G, Rothhammer V, et al. Control of tumor-associated macrophages and $\mathrm{T}$ cells in glioblastoma via AHR and CD39. Nat Neurosci 2019; 22: 1533. 

expressions in human tumor-associated microglia/macrophages and monocyte-derived macrophages. Cancer Biol Ther 2015; 16: 1205-1213.

62 Liu PS, Wang HP, Li XY, et al. $\alpha$-Ketoglutarate orchestrates macrophage activation through metabolic and epigenetic reprogramming. Nat Immunol 2017; 18: 985-994.

63 Casazza A, Laoui D, Wenes M, et al. Impeding macrophage entry into hypoxic tumor areas by Sema3A/Nrp1 signaling blockade inhibits angiogenesis and restores antitumor immunity. Cancer Cell 2013; 24: 695-709.

64 Fultang L, Gamble LD, Gneo L, et al. Macrophage-derived IL1 beta and TNF alpha regulate arginine metabolism in neuroblastoma. Cancer Res 2019; 79: 611-624.

65 Kuo CY, Ann DK. When fats commit crimes: fatty acid metabolism, cancer stemness and therapeutic resistance. Cancer Commun 2018; 38: 47.

66 Zhang Y, Daquinag AC, Amaya-Manzanares F, et al. Stromal progenitor cells from endogenous adipose tissue contribute to pericytes and adipocytes that populate the tumor microenvironment. Cancer Res 2012; 72: 5198-5208.

67 Gilligan MM, Gartung A, Sulciner ML, et al. Aspirin-triggered proresolving mediators stimulate resolution in cancer. Proc Natl Acad Sci USA 2019; 116: 6292-6297.

68 Zhang Y, Sun Y, Rao E, et al. Fatty acid-binding protein E-FABP restricts tumor growth by promoting IFN-beta responses in tumor-associated macrophages. Cancer Res 2014; 74: 2986-2998.

69 Cianciaruso C, Beltraminelli T, Duval F, et al. Molecular profiling and functional analysis of macrophage-derived tumor extracellular vesicles. Cell Rep 2019; 27: 3062-3080 e3011.

70 Daurkin I, Eruslanov E, Stoffs T, et al. Tumor-associated macrophages mediate immunosuppression in the renal cancer microenvironment by activating the 15-lipoxygenase-2 pathway. Cancer Res 2011; 71: 6400-6409.

71 Nosaka T, Baba T, Tanabe Y, et al. Alveolar macrophages drive hepatocellular carcinoma lung metastasis by generating leukotriene B4. J Immunol 2018; 200: 1839-1852.

72 Hall Z, Ament Z, Wilson CH, et al. Myc expression drives aberrant lipid metabolism in lung cancer. Cancer Res 2016; 76: 4608-4618.

73 Lukic A, Wahlund CJE, Gomez C, et al. Exosomes and cells from lung cancer pleural exudates transform LTC4 to LTD4, promoting cell migration and survival via CysLT1. Cancer Lett 2019; 444: 1-8.

74 Wu H, Han Y, Rodriguez Sillke Y, et al. Lipid droplet-dependent fatty acid metabolism controls the immune suppressive phenotype of tumor-associated macrophages. EMBO Mol Med 2019; 11: e10698.

75 Su P, Wang Q, Bi E, et al. Enhanced lipid accumulation and metabolism are required for the differentiation and activation of tumor-associated macrophages. Cancer Res 2020; 80: 1438-1450.

76 Schumann T, Adhikary T, Wortmann A, et al. Deregulation of PPAR beta/delta target genes in tumor-associated macrophages by fatty acid ligands in the ovarian cancer microenvironment. Oncotarget 2015; 6: 13416-13433.

77 Linton SS, Abraham T, Liao J, et al. Tumor-promoting effects of pancreatic cancer cell exosomes on THP-1-derived macrophages. PLoS One 2018; 13: e0206759.

78 Prima V, Kaliberova LN, Kaliberov S, et al. COX2/mPGES1/PGE(2) pathway regulates PD-L1 expression in tumor-associated macrophages and myeloid-derived suppressor cells. Proc Natl Acad Sci USA 2017; 114: $1117-1122$.

79 Wen Z, Liu H, Li M, et al. Increased metabolites of 5-lipoxygenase from hypoxic ovarian cancer cells promote tumor-associated macrophage infiltration. Oncogene 2015; 34: 1241-1252.

80 Schlager SI, Madden LD, Meltzer MS, et al. Role of macrophage lipids in regulating tumoricidal activity. Cell Immunol 1983; 77: 52-68.

81 Hao JQ, Yan F, Zhang YW, et al. Expression of adipocyte/macrophage fatty acid-binding protein in tumor-associated macrophages promotes breast cancer progression. Cancer Res 2018; 78: 2343-2355.

82 Niu Z, Shi Q, Zhang W, et al. Caspase-1 cleaves PPARgamma for potentiating the pro-tumor action of TAMs. Nat Commun 2017; 8: 766.

83 Odegaard JI, Ricardo-Gonzalez RR, Eagle AR, et al. Alternative M2 activation of Kupffer cells by PPAR delta ameliorates obesity-induced insulin resistance. Cell Metab 2008; 7: 496-507.

84 Liu C, Chikina M, Deshpande R, et al. Treg cells promote the SREBP1-dependent metabolic fitness of tumor-promoting macrophages via repression of CD8(+) T cell-derived interferon-gamma. Immunity 2019; 51: 381-397 e386.

85 Affara NI, Ruffell B, Medler TR, et al. B cells regulate macrophage phenotype and response to chemotherapy in squamous carcinomas. Cancer Cell 2014; 25: 809-821.

86 Vergara D, Stanca E, Guerra F, et al. $\beta$-Catenin knockdown affects mitochondrial biogenesis and lipid metabolism in breast cancer cells. Front Physiol 2017; 8: 544.

87 Pullamsetti SS, Seeger W, Savai R. Classical IL-6 signaling: a promising therapeutic target for pulmonary arterial hypertension. J Clin Invest 2018; 128: 1720-1723.

88 Olesch C, Sha WX, Angioni C, et al. MPGES-1-derived PGE2 suppresses CD80 expression on tumor-associated phagocytes to inhibit anti-tumor immune responses in breast cancer. Oncotarget 2015; 6: 10284-10296.

89 Goossens P, Rodriguez-Vita J, Etzerodt A, et al. Membrane cholesterol efflux drives tumor-associated macrophage reprogramming and tumor progression. Cell Metab 2019; 29: 1376-1389.e4.

90 Sag D, Cekic C, Wu R, et al. The cholesterol transporter ABCG1 links cholesterol homeostasis and tumour immunity. Nat Commun 2015; 6: 6354.

91 Choi YH, Yu AM. ABC transporters in multidrug resistance and pharmacokinetics, and strategies for drug development. Curr Pharm Design 2014; 20: 793-807.

92 Talekar M, Boreddy SR, Singh A, et al. Tumor aerobic glycolysis: new insights into therapeutic strategies with targeted delivery. Expert Opin Biol Ther 2014; 14: 1145-1159.

93 Wang HR, Tang YS, Fang YF, et al. Reprogramming tumor immune microenvironment (TIME) and metabolism via biomimetic targeting codelivery of Shikonin/JQ1. Nano Lett 2019; 19: 2935-2944.

94 Woo Y, Kim H, Kim KC, et al. Tumor-secreted factors induce IL-1 beta maturation via the glucose-mediated synergistic axis of mTOR and NF-kappa B pathways in mouse macrophages. PLoS One 2018; 13 : e0209653.

95 Aslam M, Ashfaq-Khan M, Qureshi MA, et al. Rapamycin and zoledronic acid synergistically repolarize tumour associated macrophages towards anti-HCC responses. J Hepatol 2017; 66: S465. 

inflammasome-p38 MAPK-NFkappaB pathways in autophagy- and p62-dependent manners. Oncotarget 2017; 8: 40817-40831.

97 Incio J, Suboj P, Chin SM, et al. Metformin reduces desmoplasia in pancreatic cancer by reprogramming stellate cells and tumor-associated macrophages. PLoS One 2015; 10: e0141392.

98 Uehara T, Eikawa S, Nishida M, et al. Metformin induces CD11b(+)-cell-mediated growth inhibition of an osteosarcoma: implications for metabolic reprogramming of myeloid cells and anti-tumor effects. Int Immunol 2019; 31: 187-198.

99 Penny HL, Sieow JL, Adriani G, et al. Warburg metabolism in tumor-conditioned macrophages promotes metastasis in human pancreatic ductal adenocarcinoma. Oncoimmunology 2016; 5: e1191731.

100 Arlauckas SP, Garren SB, Garris CS, et al. Arg1 expression defines immunosuppressive subsets of tumor-associated macrophages. Theranostics 2018; 8: 5842-5854.

101 Phillips M, Szlosarek PW. Arginine metabolism and tumour-associated macrophages. In: Tumour-associated macrophages. New York, Springer, 2012; pp. 77-90.

102 Landau BR, Laszlo J, Stengle J, et al. Certain metabolic and pharmacologic effects in cancer patients given infusions of 2-deoxy-D-glucose. J Natl Cancer Inst 1958; 21: 485-494.

103 Yen K, Travins J, Wang F, et al. AG-221, a first-in-class therapy targeting acute myeloid leukemia harboring oncogenic IDH2 mutations. Cancer Discov 2017; 7: 478-493.

104 Rohle D, Popovici-Muller J, Palaskas N, et al. An inhibitor of mutant IDH1 delays growth and promotes differentiation of glioma cells. Science 2013; 340: 626-630.

105 Altman BJ, Stine ZE, Dang CV. From Krebs to clinic: glutamine metabolism to cancer therapy. Nat Rev Cancer 2016; 16: 619-634

106 Yuneva M, Zamboni N, Oefner P, et al. Deficiency in glutamine but not glucose induces MYC-dependent apoptosis in human cells. J Cell Biol 2007; 178: 93-105.

107 Polis B, Srikanth KD, Gurevich V, et al. L-norvaline, a new therapeutic agent against Alzheimer's disease. Neural Regen Res 2019; 14: 1562-1572.

108 Locasale JW, Grassian AR, Melman T, et al. Phosphoglycerate dehydrogenase diverts glycolytic flux and contributes to oncogenesis. Nat Genet 2011; 43: 869-874.

109 Possemato R, Marks KM, Shaul YD, et al. Functional genomics reveal that the serine synthesis pathway is essential in breast cancer. Nature 2011; 476: 346-350.

110 Zhang B, Zheng A, Hydbring P, et al. PHGDH defines a metabolic subtype in lung adenocarcinomas with poor prognosis. Cell Rep 2017; 19: 2289-2303.

111 Netea MG, Dominguez-Andres J, Barreiro LB, et al. Defining trained immunity and its role in health and disease. Nat Rev Immunol 2020; 20: 375-388.

112 Corbet C, Feron O. Tumour acidosis: from the passenger to the driver's seat. Nat Rev Cancer 2017; 17: 577-593.

113 Bohn $\mathrm{T}$, Rapp S, Luther $\mathrm{N}$, et al. Tumor immunoevasion via acidosis-dependent induction of regulatory tumor-associated macrophages. Nat Immunol 2018; 19: 1319-1329.

114 Pilon-Thomas S, Kodumudi KN, El-Kenawi AE, et al. Neutralization of tumor acidity improves antitumor responses to immunotherapy. Cancer Res 2016; 76: 1381-1390.

115 Eleftheriadis T, Pissas G, Karioti A, et al. Dichloroacetate at therapeutic concentration alters glucose metabolism and induces regulatory T-cell differentiation in alloreactive human lymphocytes. J Basic Clin Physiol Pharmacol 2013; 24: 271-276.

116 Puchalska P, Huang XJ, Martin SE, et al. Isotope tracing untargeted metabolomics reveals macrophage polarization-state-specific metabolic coordination across intracellular compartments. Iscience 2018; 9: $298-313$.

117 Chaleckis R, Meister I, Zhang $\mathrm{P}$, et al. Challenges, progress and promises of metabolite annotation for LC-MS-based metabolomics. Curr Opin Biotechnol 2019; 55: 44-50.

118 Jenkins RW, Barbie DA, Flaherty KT. Mechanisms of resistance to immune checkpoint inhibitors. Br J Cancer 2018; 118: 9-16.

119 Zheng X, Weigert A, Reu S, et al. Spatial density and distribution of tumor-associated macrophages predict survival in non-small-cell lung carcinoma. Cancer Research 2020; in press [https://doi.org.10.1158/0008-5472. CAN-20-0069]. 